\title{
Intravascular Polarimetry for Tissue Characterization of Coronary Atherosclerosis
}

\author{
Kenichiro Otsuka, MD, PhD; Martin Villiger, $\mathrm{PhD}$; \\ Seemantini K. Nadkarni, PhD; Brett E. Bouma, PhD
}

\begin{abstract}
The microscopic tissue structure and organization influence the polarization of light. Intravascular polarimetry leverages this compelling intrinsic contrast mechanism by using polarization-sensitive optical frequency domain imaging to measure the polarization properties of the coronary arterial wall. Tissues rich in collagen and smooth muscle cells appear birefringent, while the presence of lipid causes depolarization, offering quantitative metrics related to the presence of important components of coronary atherosclerosis. Here, we review the basic principles, the interpretation of polarization signatures, and first clinical investigations of intravascular polarimetry and discuss how this extension of contemporary intravascular imaging may advance our knowledge and improve clinical practice in the future.
\end{abstract}

Key Words: Collagen; Intracoronary optical coherence tomography; Macrophage; Plaque composition; Polarized light; Smooth muscle cell

I ntravascular optical coherence tomography (OCT) and optical frequency domain imaging (OFDI) use infrared light to visualize the subsurface microstructure of the coronary arterial wall with unparalleled high spatial resolution (approximately $10 \mu \mathrm{m}$ and approximately $40 \mu \mathrm{m}$ in the radial and lateral directions, respectively). ${ }^{\mathbf{1 , 2}}$ Intracoronary OCT and OFDI have been well established for studying plaque morphology, ${ }^{3-6}$ guiding percutaneous coronary intervention, ${ }^{\mathbf{7}, 8}$ and monitoring vascular tissue response.9,10 Despite their high spatial resolution revealing rich structural details of atherosclerotic lesions, the scattering signal composing tomography images offers only limited insight into tissue and plaque composition,, $\mathbf{2 1 1 - 1 3}$ and consensus among expert readers on detailed plaque interpretation remains modest and qualitative. .14,15 $^{\text {There is a clinical need to }}$ advance intravascular optical imaging by providing quantitative tissue characterization for improved percutaneous therapy.

Multimodal imaging that combines OCT with additional imaging modalities offers interesting perspectives, ${ }^{\mathbf{1 1 , 1 6}}$ but requires dedicated imaging instrumentation that complicates clinical translation. Instead, we have been advancing polarization-sensitive (PS)-OFDI, which is an extension of OCT that measures the depth-dependent polarization state of the light scattered by tissue, ${ }^{17-19}$ and is closely compatible with current clinical OFDI instrumentation. Tissue with fibrillar architecture, such as interstitial collagen or arrayed smooth muscle cells (SMC), exhibits birefringence, an optical property that alters the polarization state of light propagating through these tissues, and offers a compelling intrinsic contrast mechanism. Collagen is a critical component of the arterial wall and imparts mechanical integrity to the fibrous cap, separating the lumen from an underlying necrotic core. Its synthesis by intimal SMC and degradation by matrix-metalloproteinases play a crucial role in plaque progression. ${ }^{20}$ The potential of PS-OCT to enhance the characterization of atherosclerotic lesions has been recognized early on. ${ }^{21-23}$ Using a PS-OCT microscope, Nadkarni et al quantitatively assessed birefringence in human aortic plaques ex vivo and demonstrated an association of birefringence with thick collagen fibers and the presence of SMC, substantiating the use of birefringence as a marker of plaque stability. ${ }^{23}$ Despite these promising results, translating PS imaging to catheter-based imaging compatible with a clinical setting proved challenging, notably because the polarization states transmitted through the catheter depend on its rotation, ${ }^{24}$ and because of the presence of systeminduced polarization distortions. ${ }^{25}$ We then developed a signal processing strategy that mitigates the resulting artifacts $^{26}$ and enabled, for the first time, measurement of depth-resolved birefringence through conventional intravascular OFDI catheters ex vivo ${ }^{26}$ and then in patients, ${ }^{27-29}$ besides other endoscopic and probe-based PS imaging. ${ }^{30,31}$ While the majority of PS-OCT applications leverages tissue birefringence as the contrast mechanism, PS imaging can also measure depolarization, ${ }^{32-34}$ which is related to the randomness of the detected polarization states. Depolarization complements birefringence for the polarimetric characterization of atherosclerotic tissue and was found to indicate the presence of lipid-rich plaque material and

Received October 3, 2019; accepted October 3, 2019; J-STAGE Advance Publication released online November 13, 2019

Wellman Center for Photomedicine, Massachusetts General Hospital, Harvard Medical School, Boston, MA (K.O., M.V., S.K.N., B.E.B.); Institute for Medical Engineering and Science, Massachusetts Institute of Technology, Cambridge, MA (B.E.B.), USA The first two authors contributed equally to this work (K.O., M.V.).

Mailing address: Kenichiro Otsuka, MD, PhD, Wellman Center for Photomedicine, Massachusetts General Hospital, Harvard Medical School, 40 Blossom Street, Boston, MA 02114, USA. E-mail: kotsuka@mgh.harvard.edu

ISSN-2434-0790 All rights are reserved to the Japanese Circulation Society. For permissions, please e-mail: cr@j-circ.or.jp 
A)

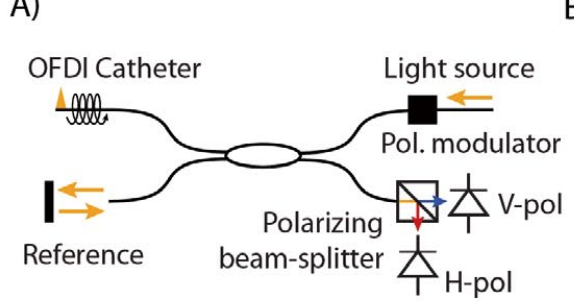

B)

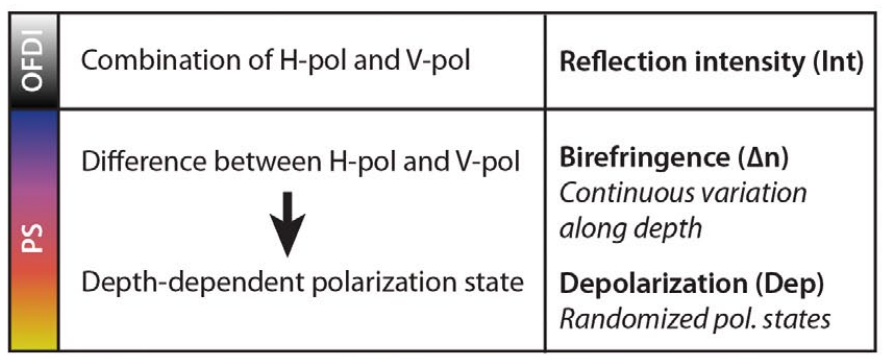

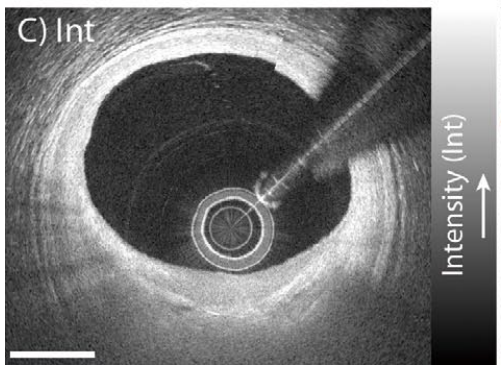
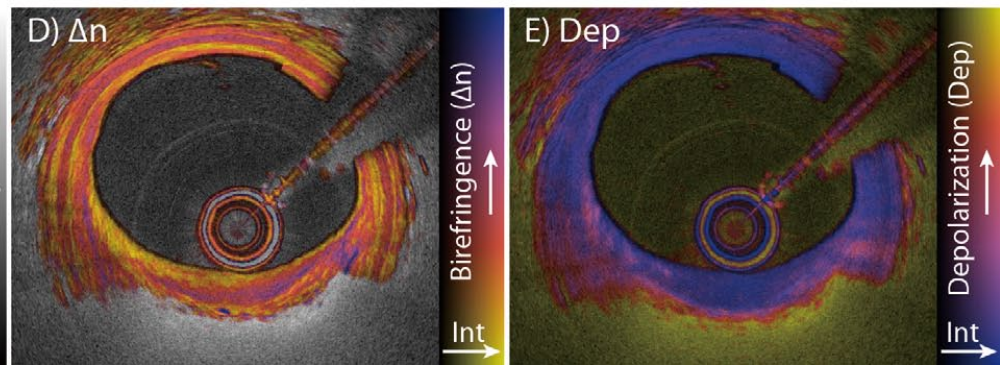

Figure 1. Working principles of intravascular polarimetry (IVP) with polarization-sensitive optical frequency domain imaging (PS-OFDI). IVP is compatible with current intravascular OFDI catheters and enables measurement of tissue polarization properties simultaneously with the conventional reflection intensity. (A) The only addition to conventional OFDI for enabling PS measurements is a polarization modulator that alternates the polarization state of the light incident on the tissue between depth scans. (B) Analyzing the spatial variation of the detected states allows reconstruction of birefringence $(\Delta n)$ and depolarization (Dep), in addition to the conventional reflection intensity image (Int). (C-E) IVP signals measured in vivo in the left anterior descending coronary artery of a 75-year-old man. (C) Intensity (Int) of the reflection signal showing subsurface plaque morphology in a conventional logarithmic gray scale. (D) Birefringence $(\Delta n)$ in color hue, overlaid on the reflection signal, indicating regions and layers of distinct birefringence. Birefringence is displayed only in areas of low depolarization. (E) Depolarization (Dep) in color hue, overlaid on the reflection signal, indicating zones of pronounced depolarization. Scale bar, $1 \mathrm{~mm}$. Reproduced with permission from Villiger et al${ }^{26}$ with permission from Elsevier.

macrophages. ${ }^{26,28}$

Intravascular polarimetry (IVP) with PS-OFDI enables quantitative and objective tissue characterization in parallel to conventional structural OFDI through conventional intravascular catheters. Here, we review the working principles, interpretation, and first clinical results of IVP by highlighting results from our ex vivo and recent clinical studies.

\section{Working Principles of PS-OFDI}

Light is an electromagnetic wave and its electric and magnetic fields oscillate in a plane orthogonal to the propagation direction of the beam. The wavelength-swept light source used for OFDI generates light with a defined polarized state, which describes the oscillation pattern of the fields in this orthogonal plane. The polarization state is affected by propagation through tissue as well as the optical fiber and components in the imaging system. The rotation of the fiber-optic probe in the imaging catheter furthermore induces dynamic changes in the transmitted polarization states. OFDI measures the interference between scattered sample light and a reference beam, but only light with identical polarization states can interfere. Because the polarization state of the sample light varies as a function of catheter rotation and tissue depth, even conventional OFDI systems use 2 detection channels with orthogonal polarization states (Figure 1). The sample light is split between the 2 channels according to its specific polarization state and all the light is detected. Tomograms of conven- tional OFDI combine the 2 channels to measure the reflection intensity as a function of depth independent of polarization effects and catheter rotation.

PS-OCT analyzes the ratio and phase difference between the signals in the 2 detection channels to recover the polarization state of the detected sample light as a function of its depth travelled into the tissue. ${ }^{19}$ To unambiguously determine the sample-induced polarization variations with catheter-based PS-OCT independent of the fiber-induced polarization changes, we modulate the polarization state of the illumination light between consecutive depth-scans, ${ }^{35}$ although recent work suggests that a single input state may suffice. ${ }^{\mathbf{3 6}}$ Tissue with fibrillar architecture, such as interstitial collagen or arrayed SMC, exhibits birefringence, which describes the difference in the tissue's refractive index between light polarized parallel to the fibrillar tissue components and light having a perpendicular polarization. The relative phase delay (retardation) between these 2 principal polarization states causes a depth-dependent variation of the detected polarization state. Using our robust reconstruction strategy that mitigates polarization artifacts, ${ }^{37} \mathrm{we}$ analyze the rate of change of the polarization states along depth to obtain a quantitative measure of depth-resolved tissue birefringence, $\Delta \mathrm{n}$.

In addition to the deterministic change of polarization with depth caused by tissue birefringence, multiple scattering and polarization-dependent scattering in tissue containing lipid and macrophage accumulations leads to a randomization of the detected polarization states. ${ }^{\mathbf{3 2 , 3 3 , 3 8}}$ Evaluating the randomization of the detected polarization states in a 


\begin{tabular}{|c|c|c|c|}
\hline Tissue type & Intensity & Birefringence & Depolarization \\
\hline Normal intima & - Highly backscattering or signal rich & $\begin{array}{l}\text { - Low to intermediate } \\
\text { - Heterogeneous }\end{array}$ & - Low \\
\hline Media & - Low backscattering or signal poor & $\begin{array}{l}\cdot \text { High } \\
\cdot \text { Homogenous }\end{array}$ & - Low \\
\hline Fibrous tissue & - Homogeneous & $\begin{array}{l}\cdot \text { High } \\
\cdot \text { Heterogeneous }\end{array}$ & - Low to intermediate \\
\hline Fibrous cap & $\begin{array}{l}\text { - Homogeneous } \\
\text { - High reflectivity } \\
\text { - Low attenuation }\end{array}$ & $\begin{array}{l}\text { - Heterogeneous } \\
\text { - Low to intermediate } \\
\text { - Thin fibrous cap exhibits spotty } \\
\text { appearance in birefringent region }\end{array}$ & - Low to intermediate \\
\hline $\begin{array}{l}\text { Lipid pool, } \\
\text { necrotic core }\end{array}$ & - High attenuation & - Moderate (to high) & - Moderate to high \\
\hline Calcification & $\begin{array}{l}\text { - Low reflectivity and attenuation } \\
\text { - Sharp edges }\end{array}$ & $\begin{array}{l}\text { - Low to intermediate, depending on } \\
\text { underlying tissue components }\end{array}$ & $\begin{array}{l}\text { - Low to high, depending on } \\
\text { underlying tissue components }\end{array}$ \\
\hline Macrophage & $\begin{array}{l}\text { - Signal-rich, distinct, bright spots, or } \\
\text { confluent punctate regions that exceed } \\
\text { the intensity of background speckle noise }\end{array}$ & - Low to intermediate & - Moderate \\
\hline Cholesterol crystal & - Thin and linear regions of high intensity & - High & - Moderate to high \\
\hline $\begin{array}{l}\text { Thrombus } \\
\text { (white thrombus) }\end{array}$ & $\begin{array}{l}\text { - Medium reflectivity } \\
\text { - Low attenuation }\end{array}$ & - Low & - Very low \\
\hline
\end{tabular}

PS-OFDI, polarization-sensitive optical frequency domain imaging.

small neighborhood around each pixel, we express depolarization as the ratio of the depolarized signal intensity to the total signal intensity (complement to 1 of the degree of polarization [1-degree of polarization $]^{34}$ ). Depolarization ranges from 0 for completely polarized light without any randomness to one for completely random polarization states. In addition to tissue-induced depolarization, regions where the OFDI signal falls to the noise floor also appear depolarizing.

To provide a convenient visualization of the complementary information available with the polarimetric analysis and the conventional reflection signal, we use a color map that encodes the polarization metric as color hue and the conventional reflection signal as brightness (Figure 1). Birefringence maps display the range $0-1.8 \times 10^{-3}$, and depolarization maps, the range $0-0.5$. For maps of tissue birefringence, we further mask areas of increased depolarization, where the randomization of the polarization states frustrates a reliable measure of tissue birefringence, by simply displaying the conventional grayscale reflection tomogram.

\section{Tissue Characterization With IVP}

Figure 1C-E shows IVP of a fibroatheroma in the left anterior descending coronary artery in a 75-year-old male patient. The birefringence map enhances many features that are already perceptible in the conventional OFDI reflection image. The tunica media, for instance, appears as a pinkish band with relatively high birefringence, often bordered by fine yellow lines of low birefringence at the locations of the internal and external elastic laminae. In comparison, the contrast between the intimal tissue and the media in conventional tomograms is less pronounced. Additionally, the birefringence can vary across tissue layers that appear homogeneous in conventional tomograms, providing complementary insight. In Figure 1E, the fibrous cap exhibits a gradient of low to higher birefringence across its cap thickness in the area of 6-8 o'clock. The depolarization map indicates low depolarization throughout the full thickness of the vessel wall in areas of minimal disease. Slight depolarization is noticeable at the shoulders of the cap close to the lumen, and abrupt strong depolarization occurs in the lipid-rich plaque material below the fibrous cap.

While the polarization metrics enable quantitative analysis and reveal intrinsic optical properties of the tissue, there are several tissue components that contribute to the observable polarization effects. In order to investigate their respective contributions, we compared IVP of human cadaveric coronary arteries with matching histology. ${ }^{26}$ The main findings are summarized in Table, detailing the identified polarization properties along with their corresponding OFDI intensity features. Figure 2 displays an overview of characteristic tissue features of coronary atherosclerosis, showing both IVP images and matching histology. ${ }^{26}$ Quantitative analysis of segmented tissue types showed that the media featured the highest birefringence, followed by intimal regions containing fibrous tissue. Consistent with a previous microscopy PS-OCT study, ${ }^{23}$ our observation suggests that collagen fibers and SMC content are the main contributors to the birefringence measured with IVP. The highest depolarization was observed in advanced lesions showing lipid/ necrotic core. This clear depolarization signature may improve the identification of fibroatheromas, for which conventional OFDI lacks specificity. ${ }^{39,40} \mathrm{We}$ speculate that lipid droplets exceeding the size of the wavelength used for OFDI and small cholesterol crystals (CC) are the origin of the observed depolarization. Furthermore, Figure 2 shows PS-OFDI examples of calcification, CC, and macrophages with matching histology. With IVP, calcifications exhibit low birefringence and low depolarization when embedded in fibrous tissue but appear more depolarizing in lipid-rich lesions. The OFDI reflection signal shows CC as thin, linear regions of high intensity, frequently found in the fibrous cap covering a necrotic core or within the necrotic core. ${ }^{1}$ $\mathrm{CC}$ identified on conventional OFDI have been implicated in plaque vulnerability. ${ }^{41}$ Consistent with the known birefringence property and dimensions of $\mathrm{CC}$, plaque regions containing small disordered $\mathrm{CC}$ depolarize and areas with 

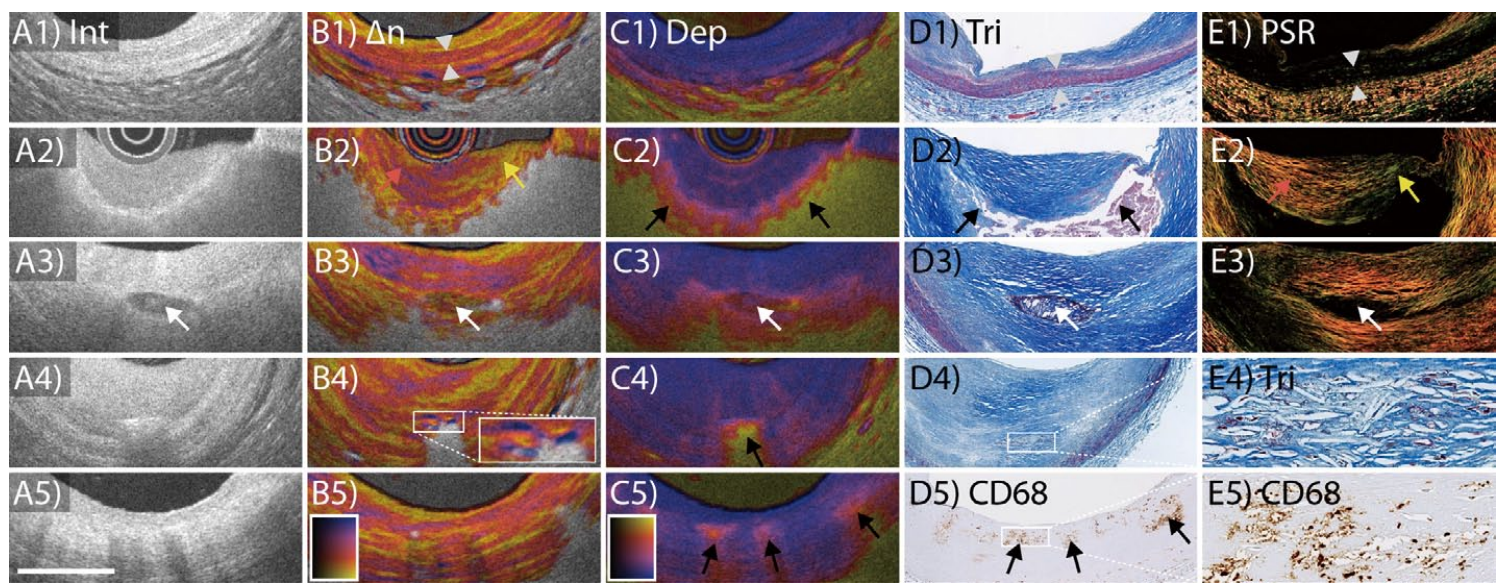

Figure 2. Polarization-sensitive optical frequency domain imaging (PS-OFDI) of (A1-E1) normal artery, (A2-E2) fibroatheroma, (A3-E3) fibrocalcific plaque, (A4-E4) cholesterol crystals, and (A5-E5) macrophage accumulations with matching histology. Scale bar, $1 \mathrm{~mm}$. $\Delta \mathrm{n}$, birefringence; Dep, depolarization; Int, intensity; PSR, Picrosirius red staining; Tri, trichrome staining. Adopted from Villiger et al26 with permission from Elsevier.

larger or aligned $\mathrm{CC}$ additionally cause a birefringence signature. ${ }^{26}$ In reflection images confusion with microcalcifications is possible, ${ }^{\mathbf{4 2}}$ and the additional polarization signatures may improve the objective identification of CC that has been speculated to be associated with rupture of the fibrous cap. Last, in conventional OFDI, macrophage accumulations in the fibrous cap cause signal-rich, distinct, bright spots, or confluent punctate regions. ${ }^{1}$ In IVP, superficial regions with macrophage accumulations were associated with subtle depolarization, which may aid in the automatic detection of macrophages by providing a quantitative metric.

\section{Repeatability of Polarimetry}

Motivated by the promising results from the cadaver study, we conducted the first in-human pilot study of IVP at the Erasmus University Medical Center in Rotterdam, the Netherlands. ${ }^{27,28,43}$ Given that IVP is an extension of commercial OFDI (FastView ${ }^{\mathrm{TM}}$; Terumo, Tokyo, Japan) used in clinical practice, the PS-OFDI procedure is identical to that of conventional OFDI from the operator's perspective. Given the promise of quantitative and objective polarization metrics and considering the previous struggle with artifactual PS images, the principal goal of this first study was to assess the robustness of IVP in a clinical setting. We evaluated the repeatability of the measured polarization properties by comparing repeat IVP pullbacks performed in 30 patients. Pearson correlation analysis indicated best agreement for the birefringence measurements, followed by the gray value of the reflection signal. Depolarization, with a correlation coefficient of 0.78 , was the poorest, but still had very acceptable agreement. ${ }^{43}$ This important finding indicates that the polarization properties measured with IVP are sufficiently robust and reliable to serve for studying the polarization properties of coronary atherosclerosis in a clinical setting.

\section{Polarimetry of Distinct Plaque Morphologies}

After validating the repeatability of IVP measurements, we investigated the polarization metrics evaluated in the entire tunica intima of individual cross-sections between different plaque types, as classified using conventional OFDI morphological features, in the 30 patients imaged during the first-in-human pilot study (acute coronary syndrome [ACS], $\mathrm{n}=12$; stable angina pectoris [SAP], $\mathrm{n}=18) .{ }^{28}$ Consistent with the previous histopathology ${ }^{26}$ fibrous plaques had the highest birefringence. We observed that plaque birefringence is reduced in plaque subtypes that contain more lipid, while depolarization becomes more pronounced with the presence of lipid/necrotic core material. ${ }^{28}$

In the same study, we also confirmed that calcifications in fibrous regions have lower birefringence than those in lipid-rich regions. ${ }^{28}$ Calcification is closely related to disease burden in coronary atherosclerosis and serves as a common surrogate marker. ${ }^{\mathbf{4 4}, \mathbf{4 5}}$ Whereas small calcium deposits in coronary arteries have been shown to be associated with the presence of fibroatheromas, dense calcifications are a hallmark of stable plaques. ${ }^{44}{ }^{46}$ Further research is warranted to investigate whether the polarization features of calcifications measured with IVP offer further insight into their association with plaque vulnerability.

\section{Polarization Features of Fibrous Caps in ACS and SAP Lesions}

We further evaluated the polarization properties locally in the fibrous caps of culprit lesions in patients with ACS and/or plaque rupture (PR) and compared them with the caps in SAP patients. Figure 3 shows the results of this analysis, together with a representative IVP example of each patient group. We observed that fibrous caps in ACS/PR patients had significantly lower birefringence than in SAP patients. ${ }^{28}$ This is consistent with histopathology, ${ }^{47}$ suggesting that ACS fibrous caps lack thick collagen fibers and layered SMC. Thick collagen fibers impart mechanical strength to fibrous caps ${ }^{48}$ and birefringence may serve as a 
(A)

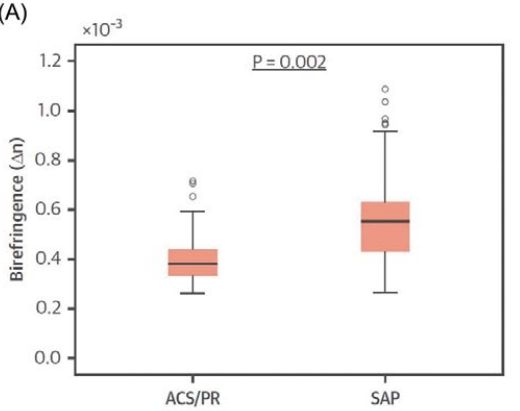

(B)

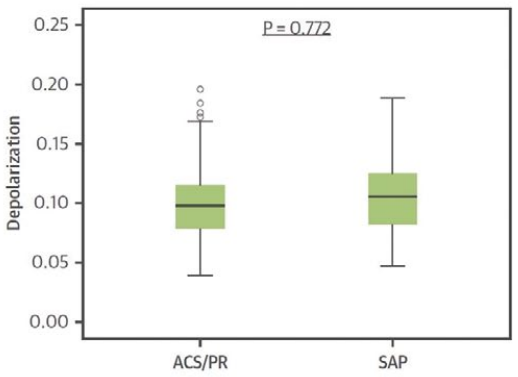

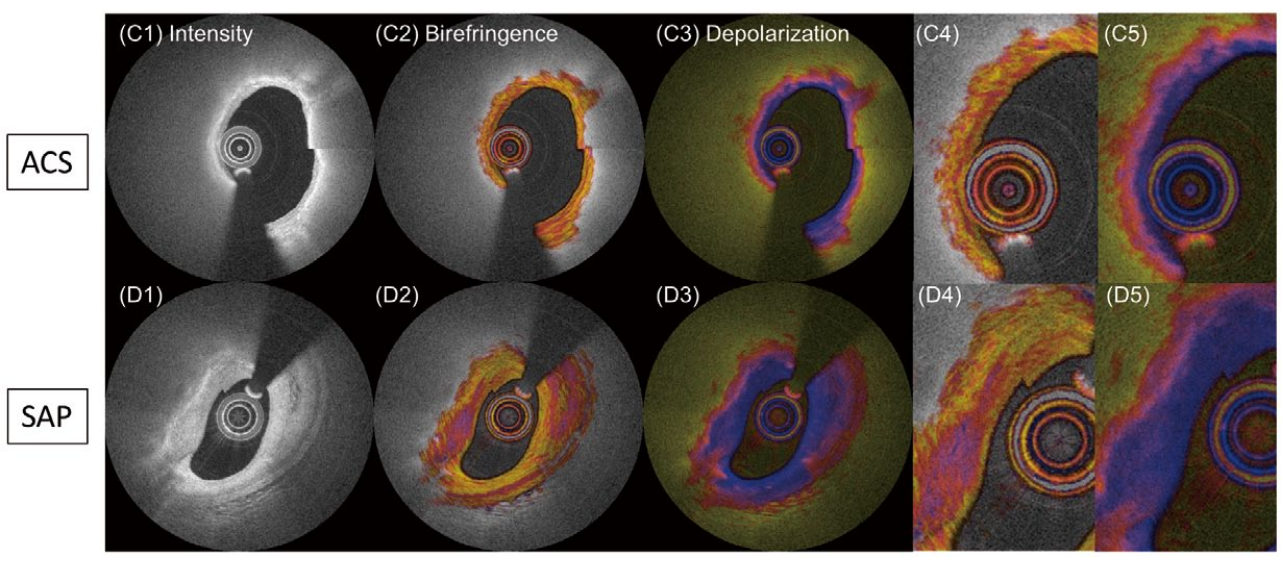

Figure 3. (A) Birefringence and (B) depolarization of fibrous caps in patients with (C1-C5) acute coronary syndrome/plaque rupture (ACS/PR) and (D1-D5) stable angina pectoris (SAP). Adopted from Otsuka et al ${ }^{\mathbf{2 8}}$ with permission from Elsevier.

biomarker of fibrous cap stability, complementing fibrous cap thickness and structural biomechanical stress. ${ }^{49}$ It is important to note that the influence of microcalcifications on the polarization properties remains unknown. Derived mainly from the apoptosis of SMC,47 these microscopic structures $(5-15 \mu \mathrm{m})$ can be visualized only with the higher resolution of $\mu \mathrm{OCT}$, which is not currently available in a clinical setting. ${ }^{50}$ Microcalcifications may exacerbate the reduction of birefringence in fibrous caps caused by their diminished collagen content.

Furthermore, histopathological studies have demonstrated that not only fibrous cap thickness but also the presence of inflammatory macrophages is an important determinant of PR. ${ }^{51}$ Analyzing the polarization properties of the thinnest part of the fibrous caps, we observed that depolarization was positively correlated with clinical presentation as ACS/ PR and with the normalized standard deviation, an imaging metric derived from conventional OFDI reflection images that has been shown to correlate with macrophage content. ${ }^{52,53}$ Depolarization may help in the identification of macrophage accumulation within fibrous caps. Additionally, the pronounced depolarization caused by the necrotic core below the fibrous cap may offer an objective delineation of the cap border and assessment of cap thickness, which remains highly variable when assessed manually by expert OCT readers. ${ }^{15}$

\section{ACS Without PR}

The increase in prevalence of ACS caused by plaque erosion is attracting attention for improving diagnosis, management, and therapy of this second leading cause of ACS.,54 Although definite diagnosis of plaque erosion in vivo remains challenging, clinical OCT studies suggest that ACS culprit lesions caused by plaque erosion exhibit few "vulnerable" features compared with those caused by PR. ${ }^{47}$ Histopathological studies have reported that eroded plaques generally correspond to negatively remodeled lesions having activated SMC embedded in a proteoglycanrich matrix consisting primarily of collagen type III, hyaluronan, and versican, ${ }^{47}$ which would be expected to cause increased birefringence. In the pilot study of IVP, however, we observed that the fibrous caps of both ACS etiologies (i.e., ruptured and non-ruptured plaques) had lower birefringence than caps in target lesions of SAP patients. Further study of the polarization properties of plaque erosion is warranted to complement current efforts of assessing endothelial shear stress and function, and other factors related to plaque erosion..$^{55,56}$

\section{Polarization Features of Coronary Thrombus}

Thrombus formation and its remodeling have been implicated in the rapid progression of coronary artery stenosis. ${ }^{6}$ In the pilot study of IVP, we reported that the birefringence and depolarization of white thrombus was very low. ${ }^{28} \mathrm{We}$ did not observe massive red thrombus, which frequently occurs in the culprit lesion of ST-segment elevation myocardial infarction. Figure 4 shows IVP images of ACS culprit lesions, where fresh thrombus exhibits very low 

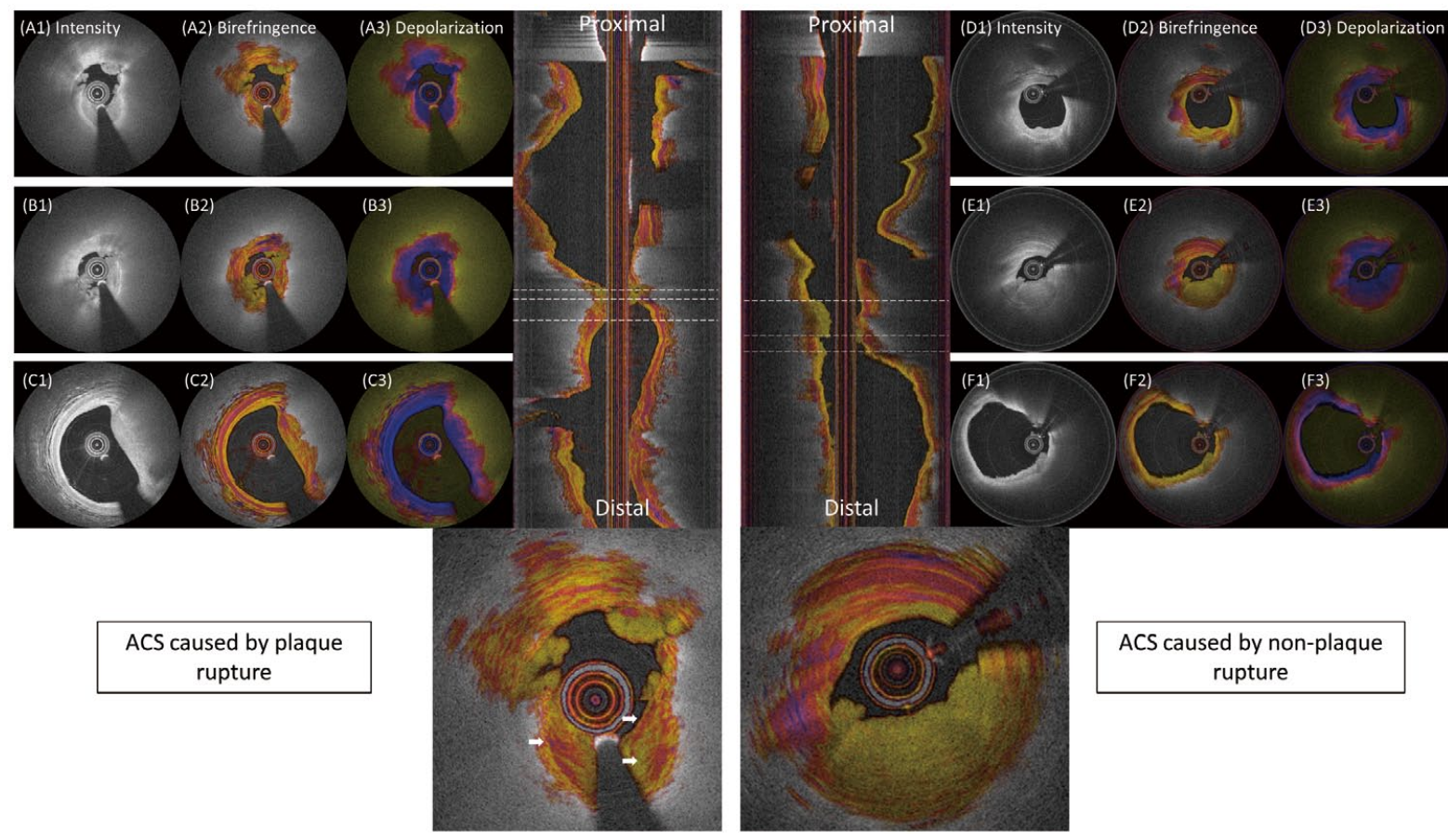

Figure 4. Birefringence and depolarization in acute coronary syndrome (ACS) culprit lesions according to plaque rupture status. $(\mathbf{A}, \mathbf{B})$ Intravascular polarimetry (IVP) of plaque rupture and white thrombus. (B2) Regions with intermediate to high birefringence indicate a higher degree of tissue organization. (D-F) IVP of ACS culprit lesions without plaque rupture. Adopted from Otsuka et $\mathrm{a}^{\mathbf{2 8}}$ with permission from Elsevier.

birefringence. We speculate that thrombus areas with a higher birefringence (white arrows) correspond to an earlier thrombus that is already undergoing remodeling and exhibits a higher degree of organization..$^{27}$ IVP may be able to assess and age thrombus and characterize the remodeling involved in the healing process, including the recruitment of SMC and the initial deposition of collagen type III, to be replaced later by collagen type I. ${ }^{47}$

\section{Future Perspectives}

Additional histopathological studies of coronary arteries are warranted to detail the specific contribution of SMC and collagen to the birefringence signal and also investigate the impact of proteoglycans and elastin. The precise physical mechanism and the compositional dependence of the observed depolarization signature should also be better understood. Additional in vitro or animal studies investigating the polarization signatures of coronary thrombosis, its organization, and the ensuing vascular healing are necessary to refine our interpretation of these signals in the clinical setting. Eventually, prospective studies in larger populations are needed to evaluate how IVP could help to improve patient outcomes and optimize medical therapy. IVP may also serve to assist drug trials by evaluating collagen and lipid content of coronary plaques as surrogate metrics to clinical outcome in response to new drugs that target atherosclerosis.

In a further technical development, we extended our reconstruction method to recover not only the scalar amount of birefringence, but also its optic axis orientation as a function of depth in the arterial wall. ${ }^{57}$ The optic axis indicates the orientation of the fibrillar tissue components in a plane orthogonal to the beam axis. Intimal tissue with minimal disease was found to feature a longitudinal optic axis orientation, while the tunica media aligns circumferentially. We speculate that the optic axis metric can provide additional insight into the organization process of thrombus and the vascular healing response following ACS or stent implantation.

The vascular response to stent implantation is of particular importance for assessing the risk of stent failure in patients. ${ }^{58}$ In a recent swine study investigating vascular response to the implantation of bioresorbable vascular scaffolds, polarization properties measured with IVP reflected tissue organization and inflammation, corresponding to SMC alignment as well as macrophage accumulation in the neointima..$^{59}$ IVP of neointima in patients is likely to advance our understanding of neoatherosclerosis and stent thrombosis and could lead to improved risk stratification and refined medical therapy.

Thus far, our studies have demonstrated that IVP provides access to a powerful source of image contrast, and visualizes structural and compositional features that are imperceptible in the conventional reflection tomograms. Yet, our studies have merely revealed differences in polarization properties between manually segmented tissue types or plaque regions. Combined, the quantitative polarization metrics and the conventional tomograms define multidimensional signals with an enhanced feature space that is ideally suited for automated segmentation and lesion classification by leveraging the powerful capability of 
advanced machine learning and deep neural networks. ${ }^{60}$ Automated segmentation and classification would be enabling both for facilitating the use of intravascular OCT in clinical practice and for improving on the modest interobserver/institution agreements found for OCT/OFDI measurements. ${ }^{14,15}$

\section{Conclusions}

Improved reconstruction methods have enabled IVP of the coronary arterial wall in patients, providing quantitative measurements of tissue birefringence and depolarization, in parallel with conventional OFDI imaging. From a practical perspective, IVP is performed in a manner identical to conventional OFDI, uses the same catheters, and imposes no additional constraint on the imaging procedure. Birefringence offers insight into the collagen and SMC content, which are important determinants of plaque stability. Depolarization highlights the presence of lipid and macrophages. These polarization metrics may improve automated identification and classification of atherosclerotic lesions and enable automated measurement, for example, of fibrous cap thickness, which would facilitate the practical clinical use of intravascular imaging. Additionally, IVP has significant potential as a research instrument, for investigating coronary plaque composition and stability, characterizing in-stent neoatherosclerosis, or in the testing of new drugs.

\section{Funding}

This work was supported by the National Institutes of Health (grants P41EB-015903 and R01HL-119065) and by Terumo Corporation. B.E.B. was supported in part by the Professor Andries Querido visiting professorship of the Erasmus University Medical Center in Rotterdam.

\section{Disclosures}

Massachusetts General Hospital and the Erasmus University Medical Center have patent licensing arrangements with Terumo Corporation. B.E.B. and M.V. have the right to receive royalties as part of the licensing arrangements. The other authors declare no conflicts of interest.

\section{Acknowledgments}

K.O. acknowledges partial support from the Japan Heart Foundation/ Bayer Yakuhin Research Grant Abroad, the Uehara Memorial Foundation Postdoctoral Fellowship, and the Japan Society for the Promotion of Science Overseas Research Fellowship.

\section{References}

1. Tearney GJ, Regar E, Akasaka T, Adriaenssens T, Barlis P, Bezerra $\mathrm{HG}$, et al. Consensus standards for acquisition, measurement, and reporting of intravascular optical coherence tomography studies: A report from the International Working Group for Intravascular Optical Coherence Tomography Standardization and Validation. J Am Coll Cardiol 2012; 59: 1058-1072.

2. Bouma BE, Villiger M, Otsuka K, Oh WY. Intravascular optical coherence tomography [Invited]. Biomed Opt Express 2017; 8: $2660-2686$.

3. Iguchi T, Hasegawa T, Otsuka K, Matsumoto K, Yamazaki T, Nishimura $\mathrm{S}$, et al. Insulin resistance is associated with coronary plaque vulnerability: Insight from optical coherence tomography analysis. Eur Heart J Cardiovasc Imaging 2014; 15: 284-291.

4. Jia H, Dai J, Hou J, Xing L, Ma L, Liu H, et al. Effective anti-thrombotic therapy without stenting: Intravascular optical coherence tomography-based management in plaque erosion (the EROSION study). Eur Heart J 2017; 38: 792-800.

5. Jackson R, Al-Hussaini A, Joseph S, van Soest G, Wood A, Macaya F, et al. Spontaneous coronary artery dissection: Pathophysiological insights from optical coherence tomography. JACC Cardiovasc Imaging, doi:10.1016/j.jcmg.2019.01.015.
6. Fracassi F, Crea F, Sugiyama T, Yamamoto E, Uemura S, Vergallo R, et al. Healed culprit plaques in patients with acute coronary syndromes. J Am Coll Cardiol 2019; 73: 2253-2263.

7. Räber L, Mintz GS, Koskinas KC, Johnson TW, Holm NR, Onuma Y, et al. Clinical use of intracoronary imaging. Part 1: Guidance and optimization of coronary interventions. An expert consensus document of the European Association of Percutaneous Cardiovascular Interventions. Eur Heart J 2018; 39: 3281-3300.

8. Johnson TW, Räber L, di Mario C, Bourantas C, Jia H, Mattesini A, et al. Clinical use of intracoronary imaging. Part 2: Acute coronary syndromes, ambiguous coronary angiography findings, and guiding interventional decision-making: An expert consensus document of the European Association of Percutaneous Cardiovascular Interventions. Eur Heart J 2019; 40: 2566-2584.

9. Räber L, Koskinas KC, Yamaji K, Taniwaki M, Roffi M, Holmvang L, et al. Changes in coronary plaque composition in patients with acute myocardial infarction treated with high-intensity statin therapy (IBIS-4): A serial optical coherence tomography study. JACC Cardiovasc Imaging 2019; 12: 1518-1528.

10. Ozaki Y, Garcia-Garcia HM, Beyene SS, Hideo-Kajita A, Kuku $\mathrm{KO}$, Kolm P, et al. Effect of statin therapy on fibrous cap thickness in coronary plaque on optical coherence tomography: Review and meta-analysis. Circ J 2019; 83: 1480-1488.

11. Suter MJ, Nadkarni SK, Weisz G, Tanaka A, Jaffer FA, Bouma $\mathrm{BE}$, et al. Intravascular optical imaging technology for investigating the coronary artery. JACC Cardiovasc Imaging 2011; 4: $1022-1039$.

12. Karanasos A, Simsek C, Gnanadesigan M, Van Ditzhuijzen NS, Freire R, Dijkstra J, et al. OCT assessment of the long-term vascular healing response 5 years after everolimus-eluting bioresorbable vascular scaffold. J Am Coll Cardiol 2014; 64: 2343-2356.

13. Tarkin JM, Dweck MR, Evans NR, Takx RAP, Brown AJ, Tawakol A, et al. Imaging atherosclerosis. Circ Res 2016; 118: $750-769$.

14. Gerbaud E, Weisz G, Tanaka A, Kashiwagi M, Shimizu T, Wang L, et al. Multi-laboratory inter-institute reproducibility study of IVOCT and IVUS assessments using published consensus document definitions. Eur Heart J Cardiovasc Imaging 2016; 17: $756-764$.

15. Kini AS, Vengrenyuk Y, Yoshimura T, Matsumura M, Pena J, Baber U, et al. Fibrous cap thickness by optical coherence tomography in vivo. J Am Coll Cardiol 2017; 69: 644-657.

16. Bourantas CV, Jaffer FA, Gijsen FJ, Van Soest G, Madden SP, Courtney BK, et al. Hybrid intravascular imaging: Recent advances, technical considerations, and current applications in the study of plaque pathophysiology. Eur Heart $J$ 2017; 38: 400412.

17. Hee MR, Huang D, Swanson EA, Fujimoto JG. Polarizationsensitive low-coherence reflectometer for birefringence characterization and ranging. J Opt Soc Am B 1992; 9: 903-908.

18. de Boer JF, Milner TE, van Gemert MJ, Nelson JS. Twodimensional birefringence imaging in biological tissue by polarization-sensitive optical coherence tomography. Opt Lett 1997; 22: 934-936.

19. de Boer JF, Hitzenberger CK, Yasuno Y. Polarization sensitive optical coherence tomography: A review [Invited]. Biomed Opt Express 2017; 8: $1838-1873$.

20. Libby P. Mechanisms of acute coronary syndromes and their implications for therapy. N Engl J Med 2013; 368: 2004-2013.

21. Giattina SD, Courtney BK, Herz PR, Harman M, Shortkroff S, Stamper DL, et al. Assessment of coronary plaque collagen with polarization sensitive optical coherence tomography (PS-OCT). Int J Cardiol 2006; 107: 400-409.

22. Kuo WC, Chou NK, Chou C, Lai CM, Huang HJ, Wang SS, et al. Polarization-sensitive optical coherence tomography for imaging human atherosclerosis. Appl Opt 2007; 46: 2520-2527.

23. Nadkarni SK, Pierce MC, Park BH, de Boer JF, Whittaker P, Bouma BE, et al. Measurement of collagen and smooth muscle cell content in atherosclerotic plaques using polarization-sensitive optical coherence tomography. J Am Coll Cardiol 2007; 49: $1474-1481$.

24. Pierce M, Shishkov M, Park B, Nassif N, Bouma B, Tearney G, et al. Effects of sample arm motion in endoscopic polarizationsensitive optical coherence tomography. Opt Express 2005; 13: $5739-5749$.

25. Villiger M, Zhang EZ, Nadkarni S, Oh WY, Bouma BE, Vakoc BJ. Artifacts in polarization-sensitive optical coherence tomography caused by polarization mode dispersion. Opt Lett 2013; 38: 923-925.

26. Villiger M, Otsuka K, Karanasos A, Doradla P, Ren J, Lippok 
$\mathrm{N}$, et al. Coronary plaque microstructure and composition modify optical polarization. JACC Cardiovasc Imaging 2018; 11: 16661676.

27. van der Sijde JN, Karanasos A, Villiger M, Bouma BE, Regar E. First-in-man assessment of plaque rupture by polarizationsensitive optical frequency domain imaging in vivo. Eur Heart $J$ 2016; 37: 1932

28. Otsuka K, Villiger M, Karanasos A, van Zandvoort LJC, Doradla P, Ren J, et al. Intravascular polarimetry in patients with coronary artery disease. JACC Cardiovasc Imaging, doi: 10.1016/j.jcmg.2019.06.015.

29. Van Zandvoort LJC, Otsuka K, Bouma BE, Daemen J. Intracoronary polarimetry of a honeycomb-like structure. EuroIntervention, doi:10.4244/EIJ-D-19-00431.

30. Villiger M, Lorenser D, McLaughlin RA, Quirk BC, Kirk RW, Bouma BE, et al. Deep tissue volume imaging of birefringence through fibre-optic needle probes for the delineation of breast tumour. Sci Rep 2016; 6: 28771

31. Adams DC, Hariri LP, Miller AJ, Wang Y, Cho JL, Villiger M, et al. Birefringence microscopy platform for assessing airway smooth muscle structure and function in vivo. Sci Transl Med 2016; 8: 359ra131.

32. Adie SG, Hillman TR, Sampson DD. Detection of multiple scattering in optical coherence tomography using the spatial distribution of Stokes vectors. Opt Express 2007; 15: 18033-18049.

33. Gotzinger E, Pircher M, Geitzenauer W, Ahlers C, Baumann B, Michels S, et al. Retinal pigment epithelium segmentation by polarization sensitive optical coherence tomography. Opt Express 2008; 16: 16410-16422.

34. Lippok N, Villiger M, Bouma BE. Degree of polarization (uniformity) and depolarization index: Unambiguous depolarization contrast for optical coherence tomography. Opt Lett 2015; 40: 3954-3957.

35. Saxer CE, de Boer JF, Park BH, Zhao Y, Chen Z, Nelson JS High-speed fiber based polarization-sensitive optical coherence tomography of in vivo human skin. Opt Lett 2000; 25: 1355-1357.

36. Xiong Q, Wang N, Liu X, Chen Si, Braganza CS, Bouma BE, et al. Constrained polarization evolution simplifies depth-resolved retardation measurements with polarization-sensitive optical coherence tomography. Biomed Opt Express 2019; 10: 5207-5222.

37. Villiger M, Zhang EZ, Nadkarni SK, Oh WY, Vakoc BJ, Bouma BE. Spectral binning for mitigation of polarization mode dispersion artifacts in catheter-based optical frequency domain imaging. Opt Express 2013; 21: 16353-16369.

38. Lippok N, Villiger M, Albanese A, Meijer EFJ, Chung K, Padera TP, et al. Depolarization signatures map gold nanorods within biological tissue. Nat Photonics 2017; 11: 583-588.

39. Phipps JE, Vela D, Hoyt T, Halaney DL, Mancuso JJ, Buja LM, et al. Macrophages and intravascular OCT bright spots: A quantitative study. JACC Cardiovasc Imaging 2015; 8: 63-72.

40. Nakano M, Yahagi K, Yamamoto H, Taniwaki M, Otsuka F, Ladich ER, et al. Additive value of integrated backscatter IVUS for detection of vulnerable plaque by optical frequency domain imaging: An ex vivo autopsy study of human coronary arteries. JACC Cardiovasc Imaging 2016; 9: 163-172.

41. Janoudi A, Shamoun FE, Kalavakunta JK, Abela GS. Cholesterol crystal induced arterial inflammation and destabilization of atherosclerotic plaque. Eur Heart J 2016; 37: 1959-1967.

42. Tearney GJ. OCT imaging of macrophages: A bright spot in the study of inflammation in human atherosclerosis. JACC Cardiovasc Imaging 2015; 8: $73-75$.

43. Villiger M, Otsuka K, Karanasos A, Doradla P, Ren J, Lippok $\mathrm{N}$, et al. Repeatability assessment of intravascular polarimetry in patients. IEEE Trans Med Imaging 2018; 37: 1618-1625.

44. Ehara S, Kobayashi Y, Yoshiyama M, Shimada K, Shimada Y, Fukuda D, et al. Spotty calcification typifies the culprit plaque in patients with acute myocardial infarction: An intravascular ultrasound study. Circulation 2004; 110: 3424-3429.

45. Criqui MH, Denenberg JO, Ix JH, McClelland RL, Wassel CL, Rifkin DE, et al. Calcium density of coronary artery plaque and risk of incident cardiovascular events. JAMA 2014; 311: 271 278.

46. Nakahara T, Dweck MR, Narula N, Pisapia D, Narula J, Strauss HW. Coronary artery calcification: From mechanism to molecular imaging. JACC Cardiovasc Imaging 2017; 10: 582- 593.

47. Yahagi K, Kolodgie FD, Otsuka F, Finn AV, Davis HR, Joner $\mathrm{M}$, et al. Pathophysiology of native coronary, vein graft, and in-stent atherosclerosis. Nat Rev Cardiol 2016; 13: 79-98.

48. Brown AJ, Teng Z, Evans PC, Gillard JH, Samady H, Bennett MR. Role of biomechanical forces in the natural history of coronary atherosclerosis. Nat Rev Cardiol 2016; 13: 210-220.

49. Doradla P, Otsuka K, Nadkarni A, Villiger M, Karanasos A, van Zandvoort LJC, et al. Biomechanical stress profiling of coronary atherosclerosis: Identifying a multifactorial metric to evaluate plaque rupture risk. JACC Cardiovasc Imaging, doi:10.1016/j.jcmg.2019.01.033.

50. Nishimiya K, Yin B, Piao Z, Ryu J, Osman H, Leung HM, et al. Micro-optical coherence tomography for endothelial cell visualization in the coronary arteries. JACC Cardiovasc Imaging 2019; 12: $1878-1880$.

51. Narula J, Nakano M, Virmani R, Kolodgie FD, Petersen R, Newcomb R, et al. Histopathologic characteristics of atherosclerotic coronary disease and implications of the findings for the invasive and noninvasive detection of vulnerable plaques. $\mathrm{J} \mathrm{Am}$ Coll Cardiol 2013; 61: 1041-1051.

52. Tearney GJ, Yabushita H, Houser SL, Aretz HT, Jang IK, Schlendorf KH, et al. Quantification of macrophage content in atherosclerotic plaques by optical coherence tomography. Circulation 2003; 107: 113-119.

53. Di Vito L, Agozzino M, Marco V, Ricciardi A, Concardi M, Romagnoli E, et al. Identification and quantification of macrophage presence in coronary atherosclerotic plaques by optical coherence tomography. Eur Heart J Cardiovasc Imaging 2015; 16: $807-813$.

54. Dai J, Xing L, Jia H, Zhu Y, Zhang S, Hu S, et al. In vivo predictors of plaque erosion in patients with ST-segment elevation myocardial infarction: A clinical, angiographical, and intravascular optical coherence tomography study. Eur Heart J 2018; 39: 2077-2085.

55. Quillard T, Araujo HA, Franck G, Shvartz E, Sukhova G, Libby P. TLR2 and neutrophils potentiate endothelial stress, apoptosis and detachment: Implications for superficial erosion. Eur Heart $J$ 2015; 36: $1394-1404$.

56. Yamamoto E, Thondapu V, Poon E, Sugiyama T, Fracassi F, Dijkstra J, et al. Endothelial shear stress and plaque erosion: A computational fluid dynamics and optical coherence tomography study. JACC Cardiovasc Imaging 2019; 12: 374-375.

57. Villiger M, Braaf B, Lippok N, Otsuka K, Nadkarni SK, Bouma BE. Optic axis mapping with catheter-based polarization-sensitive optical coherence tomography. Optica 2018; 5: 1329-1337.

58. Taniwaki M, Windecker S, Zaugg S, Stefanini GG, Baumgartner S, Zanchin $\mathrm{T}$, et al. The association between in-stent neoatherosclerosis and native coronary artery disease progression: A long-term angiographic and optical coherence tomography cohort study. Eur Heart J 2015; 36: 2167-2176.

59. Serruys PW, Duncker DJ, Murawska M, Sorop O, van Soest G, Villiger M, et al. Neoatherosclerosis development following bioresorbable vascular scaffold implantation in diabetic and non-diabetic swine. PLoS One 2017; 12: e0183419.

60. Gessert N, Lutz M, Heyder M, Latus S, Leistner DM, Abdelwahed YS, et al. Automatic plaque detection in IVOCT pullbacks using convolutional neural networks. IEEE Trans Med Imaging 2019; 38: $426-434$. 